\title{
Piecewise Constant Aquifer Parameter Identification Recovery
}

\author{
R. S. Anderssen ${ }^{\mathrm{a}}$ and B. P. Lamichhane ${ }^{\mathrm{b}}$ \\ ${ }^{a}$ CSIRO Mathematics, Informatics and Statistics, GPO Box 664, Canberra, ACT 2601 \\ ${ }^{\mathrm{b}}$ School of Mathematical and Physical Sciences, University of Newcastle, Callaghan, NSW 2308 \\ Email: Bob.Anderssen@csiro.au
}

\begin{abstract}
For many inverse problems which arise in contributing to real-world decision-making, such as formulating policy objectives for freshwater fish health, only an indicative understanding of the global structure of the solution is all that is required for the associated decision-support. Examples include:
\end{abstract}

(i) Situations where only some linear functional of the solution is required. As noted in various publications, this occurs in quite independent situations. They include: the recovery of moments of the spheres distribution from observations of the corresponding circles distribution on random plane sections through the spheres (Jakeman and Anderssen (1975)); the evaluation of local solutions in geodesy (Bauer et al. (2007)); a strategy for sparse recovery (Lu and Pereverzev (2009)), where the required information corresponds to some substructure in a complex solution such as the wavelength vibration of a target protein molecule in an NIR spectrum (Anderssen et al. (2003)); the evaluation of linear functionals of the molecular weight distributions of polymers (Anderssen et al. (1997); Anderssen (1999)).

(ii) Where it is only necessary to recover some feature of the solutions such as whether the solution, as a function of the independent variable, is increasing, decreasing, convex or concave. Such situations arise in: the foliage angle distribution problem (Anderssen et al. (1985, 1984)); the location of some peak in the data as arises in resolution enhancement and derivative spectroscopy (Hegland and Anderssen (2005); Anderssen and Hegland (2010)); some of the situations in (i) where the required feature can be defined as a linear functional of the solution.

In aquifer parameter identification, a similar situation arises. It is often only the regional structure of and connectivity within the aquifer that is required (Blakers et al. (2011)). For such situations, a piecewiseconstant approximation of the solution is all that is required to highlight the global features of the solution. Here, we examine the utilization of such approximations for the recovery of information about the regionalized structure of an aquifer.

Here, using simulations, we examine the numerical performance of an anzatz proposed by Chow and Anderssen (1991) for the recovery of acquifer transmissivity from observational data. The clear advantage of their localization approach is that

- it removes the need to know the precise extent of the aquifer and the corresponding boundary conditions, and

- it allows the structure of the zonation within an aquifer to be explored in an iterative manner.

Simulations, with synthetic data, confirm the utility of the proposed method to determine the zonation structure within an aquifer.

Keywords: aquifer, parameter identification, piecewise constant approximations, weak formulation 


\section{THE AQUIFER PARAMETER IDENTIFICATION PROBLEM}

Much water for agriculture and human consumption comes from underground aquifers. Of the many practical issues involved with their management, knowledge about their transmissivity, zonation and connectivity is fundamental (Bear (1972); Blakers et al. (2011)) because they constrain how the water within an aquifer system should be managed to the benefit of the various users. However, from a practical perspective, only indicative estimates of them are required, as it is the qualitative structure of their zonal and regional variations that are the criteria that supports the associated decision making in the allocation of the available water resources. Validation for there only being a need for an indicative understanding of the transmissivity, zonation and connectivity within an aquifer relates directly to the fact that an aquifer system is a geological structure which tends to have a fairly uniform structure on local scales and a hetrogeneous zonation (sometimes disconnected) structure only on larger regional scales.

In the study of steady-state flow of groundwater in a confined aquifer $\Omega$, the governing equation is the following elliptic partial differential equation (Bear (1972); Yeh (1986))

$$
-\nabla \cdot(\alpha \nabla u)=f \quad \text { in } \Omega
$$

where $\Omega \subset \mathbb{R}^{d}, d \in\{1,2,3\}$, is a bounded domain, $\alpha: \Omega \rightarrow \mathbb{R}$ denotes the transmissivity coefficient, $u: \Omega \rightarrow \mathbb{R}$ is pressure and $f$ is the source term. When the source term $f$ and the coefficient $\alpha$ are given, appropriate boundary information about the solution $u$ is required so that the corresponding forward (direct) problem of determining $u$ in $\Omega$ has a unique solution. Conversely, the recovery of information about the coefficient $\alpha$, when $u$ and $f$ are given, is an inverse problem with $\alpha$ being sensitive to perturbations in $u$ and $f$. It is the recovery of $\alpha$ from measurements of $u$ and $f$, through the utilization of equation (1), that is called the "aquifer parameter identification problem".

From a practical perspective involving observational data, the challenge posed by the aquifer parameter identification problem is complicated by the fact that

- because it is an underground structure, about which surface geology can only give marginal insight, the actual shape $\Omega$ of the aquifer is unknown,

- even if $\Omega$ were known, the boundary conditions are not, and

- the spatial locations at which measurements of the changing pressure $u$ has been recorded are quite sparse.

As a direct consequence, especially when coupling this with the fact that only an indicative understanding about the value of the transmissivity, zonation and connectivity are required, the focus must be on the formulation of simple algorithms for the recovery of information from the available data.

As explained in Chow and Anderssen (1991), where the earlier use of zonation algorithms is briefly surveyed, this was the motivation behind their formulation of a linear functional methodology. Chow and Anderssen concluded, though the earlier algorithms represented useful proposals for situations where detailed accurate data are available at a large number of locations (e.g. Emsellem and de Emarsil (1971); Sun and Yeh (1985)), they were of limited utility in most practical situations.

\section{The Chow-Anderssen Ansatz}

The essential improperly posedness in the recovery of information about the transmissivity $\alpha$ from measurements of $u$ and $f$ relates to the fact that the observed pressure (piezometric head) data $u$ must be differentiated. This is clear from the structure of equation (1).

The essence of the ansatz involves four steps:

(a) the reformulation of the partial differential equation model in terms of a weak form,

(b) the application of integration by parts to move the differentiation of the observed pressure onto the analytic test function, 
(c) the localization of the resulting linear functional representation for the transmissivity to a subregion of the aquifer where the transmissivity is assumed to be constant, and

(d) the choice of the shape of the localization and the form of test function that facilitates the computational performance of the resulting algorithm.

As explained in Chow and Anderssen (1991) and Lamichhane and Anderssen (2011), in terms of the notation of equation (1) and the application of the steps (a)-(d) of the anzatz, various linear functional formulas for the estimation of $\alpha$ on the subregion $\Lambda \subset \Omega$ can be derived which depends on the assumed regularity of the solution $u$ of equation (1) and the test function $v$. The two estimates for $\alpha$ investigated here are

$$
\hat{\alpha}_{1}=\frac{\int_{\Lambda} f v d x}{\int_{\Lambda} \nabla u \cdot \nabla v d x}, \quad u \in H^{1}(\Lambda), \quad v \in H_{0}^{1}(\Lambda),
$$

(Scheme 1)

and

$$
\hat{\alpha}_{2}=-\frac{\int_{\Lambda} f v d x}{\int_{\Lambda} u \Delta v d x}, \quad u \in L^{2}(\Lambda), \quad v \in H_{0}^{2}(\Lambda) .
$$

The special form that they take is a direct exploitation of the assumption that $\alpha$ is a constant in the subregion $\Lambda$. In Scheme 1 , the evaluation of $\hat{\alpha}_{1}$ involves the differentiation of $u$, whereas, in Scheme 2 , through an integration by parts in conjunction with the regularity imposed on the test function $v$, the evaluation if $\hat{\alpha}_{2}$ does not involve a differentiation of $u$ since it has been transferred to to $v$.

\section{The Simulations}

In the simulations to test the numerical performance of the two schemes, we consider the one-dimensional version of equation (1) with $\alpha$ modelled as a constant plus a small oscillatory perturbation of frequency $k$

$$
\alpha=10+\delta(\sin (k x)+\cos (k x)) .
$$

The solution $u$ of equation (1) is assumed to have the form

$$
u=13 x^{2}+10 x+12 \cos (3 x)+\sin (4 x)+12,
$$

with the subregion $\Lambda=[2,4] \subset \Omega$. Using $\delta=0.1$ and $\delta=0.05$, we compare the performance of the two schemes.

The forcing function $f$ is computed by using the exact solution and the exact parameter in the differential equation (1). For both schemes, two different test functions are used. A simple test function for Scheme 1 is given by the quadratic bubble function

$$
v_{q}(x)=4 \frac{(x-a)(b-x)}{(b-a)^{2}}, \quad x \in[a, b] .
$$

Consequently, $v_{q} \in H^{1}(a, b)$ and it has zero boundary condition. For Scheme 2, a test function $v \in$ $H_{0}^{2}(\Lambda)$ is required. A simple test function for $v_{q s} \in H_{0}^{2}(\Lambda)$ is the quadratic B-spline. Since $H_{0}^{2}(\Lambda) \subset$ $H_{0}^{1}(\Lambda), v_{q s}$ can also be used as a test function for Scheme 1 . In addition, the popular cubic B-spline $v_{c s}$ is utilized in the implementation of Scheme 2. These three test functions, when defined on the interval $[2,4]$, are plotted in Figure 1.

As already noted, the first scheme requires the gradient of the solution $u$ to be computed, whereas the second scheme does not. Because $u$ comes from measurements, it is appropriate to use a scheme which avoids its differentiation.

In all the Figures below, the exact and estimated values of $\alpha$ are plotted for different choices of the frequency $k$. Eighty (80) equally spaced values for $k \in[0.2,40]$ have been used. In order to highlight the 

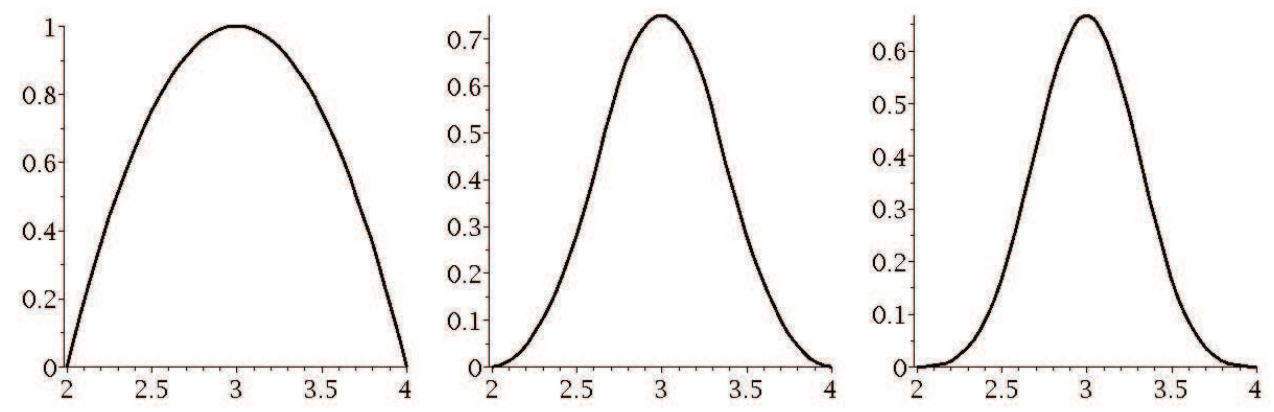

Figure 1: Test functions $v_{q}$ (left), $v_{q s}$ (middle) and $v_{c s}$ (right)
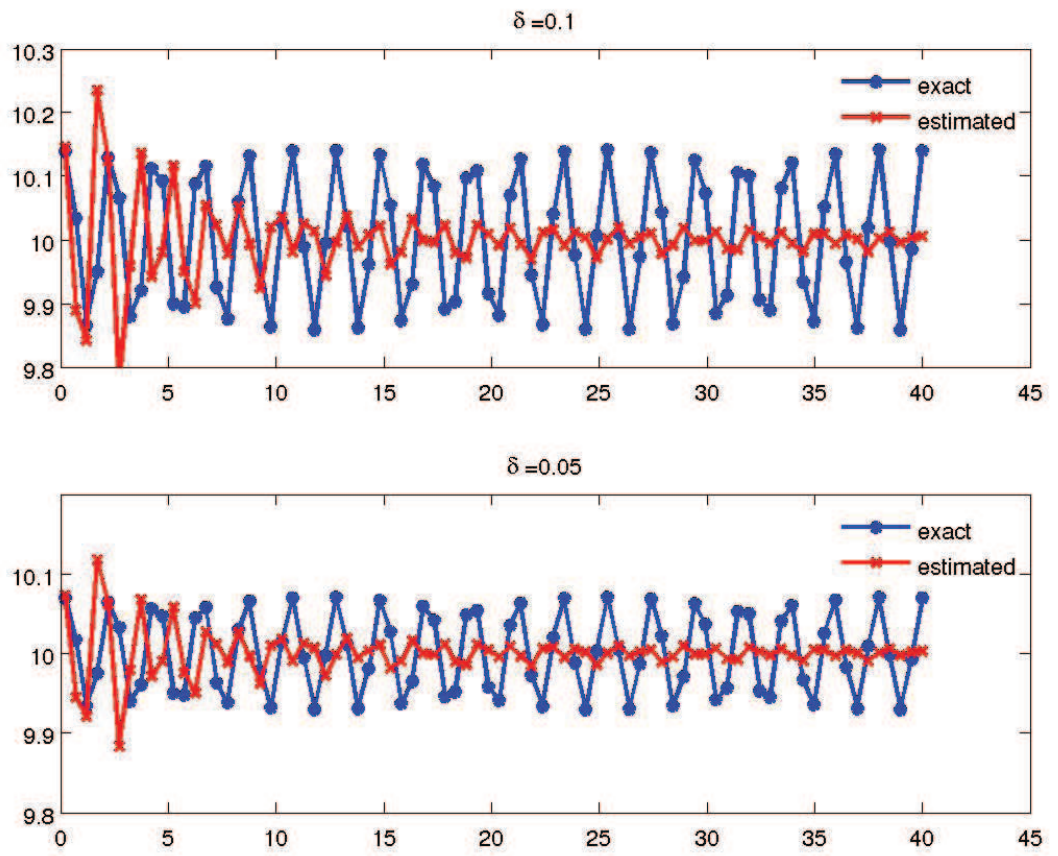

Figure 2: The exact and estimated parameter versus frequencies for $\delta=0.1$, and $\delta=0.05$ for Scheme 1 with test function $v_{q}$ 

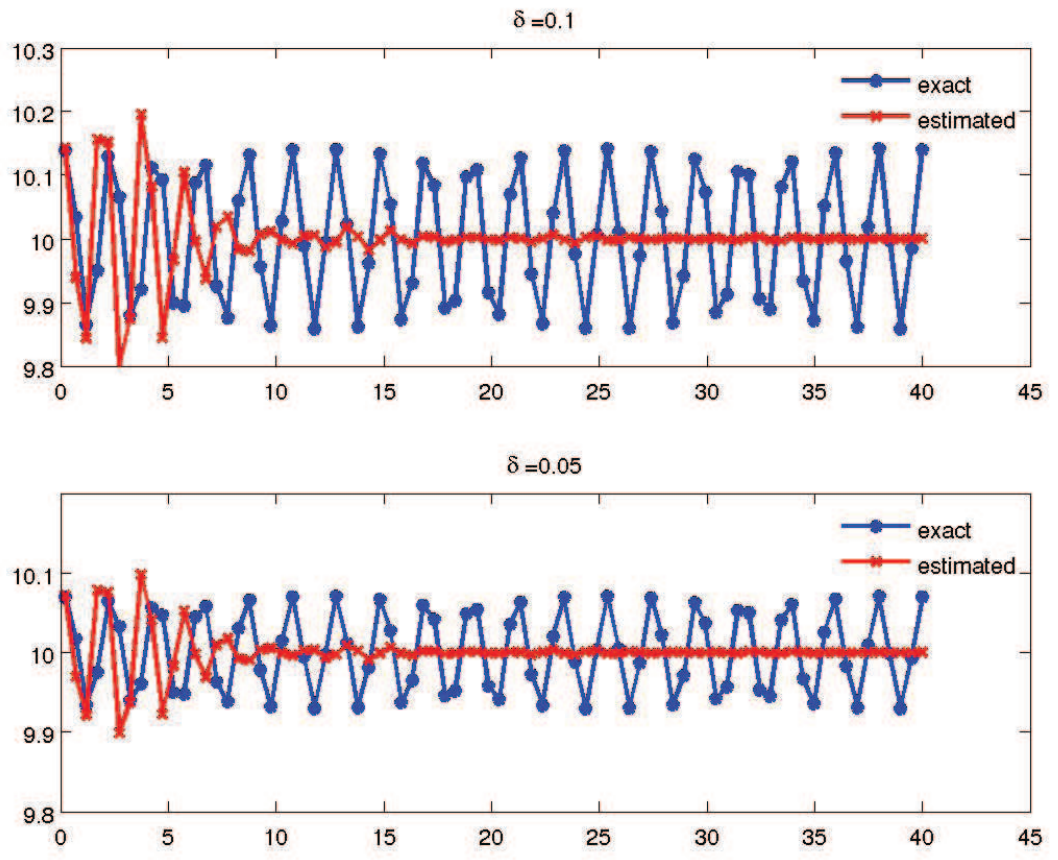

Figure 3: The exact and estimated parameter versus frequencies for $\delta=0.1$, and $\delta=0.05$ for Scheme 1 with test function $v_{q s}$

variability in the structure of $\alpha$, its value at the mid-point of the interval $[2,4]$ is plotted, for each of the discrete values of $k$.

In the two subfigures of Figure 2, the comparison of exact and estimated parameters are plotted for Scheme 1 when the test function is $v_{q}$ and $\delta=0.1$ and $\delta=0.05$, respectively. The estimated value for $\alpha$ asymptotes to the constant value 10 as the frequency $k$ increases. In addition, the errors between the exact and the estimated values are smaller for the smaller value of $\delta$.

Similarly, the corresponding comparison of the exact and estimated value of $\alpha$ for Scheme 1 , when the test function is $v_{q s}$, is plotted in two subfigures of Figure 3. The behaviour seen in Figure 2 is repeated in Figure 3. It follows that if the amplitude of the oscillatory perturbation is small, both schemes give good estimates of $\alpha$ for suitably large values of $k$. While the numerical approximations from both test functions are quite good, the convergence is better with the test function $v_{q s}$. These numerical results support the theoretical result discussed in Lamichhane and Anderssen (2011).

A comparison of the exact and estimated values of $\alpha$ for Scheme 2 are plotted in Figures 4 and 5 when the test functions $v_{q s}$ and $v_{c s}$, respectively, are used. As before, the two subfigures correspond to choosing $\delta=0.1$ and $\delta=0.05$. While the converegence for Scheme 2 is better than for Scheme 1 , it is interesting to note that both quadratic and cubic spline test functions are producing almost identical numerical estimates for Scheme 2.

\section{Conclusions}

Living with uncertainty is the nature of solving inverse problems for real-world situations such as aquifer parameter identification. From a decision making perspective, only indicative solutions are required. The exploitation of this fact is important as it represents a type of context regularization.

The utilization of the procedures outlined above for the determination of a zonation structure can be achieved by following the procedure suggested by Chow and Anderssen (1991). 

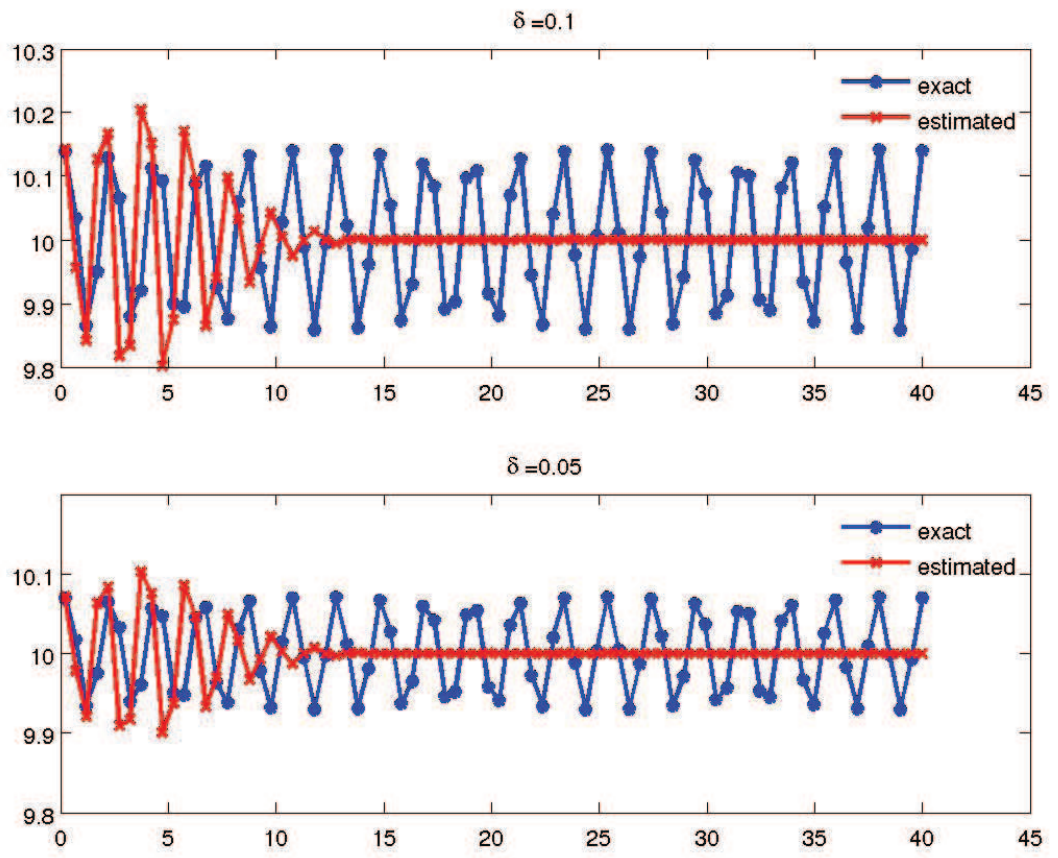

Figure 4: The exact and estimated parameter versus frequencies for $\delta=0.1$ and $\delta=0.05$ for Scheme 2 with test function $v_{q s}$
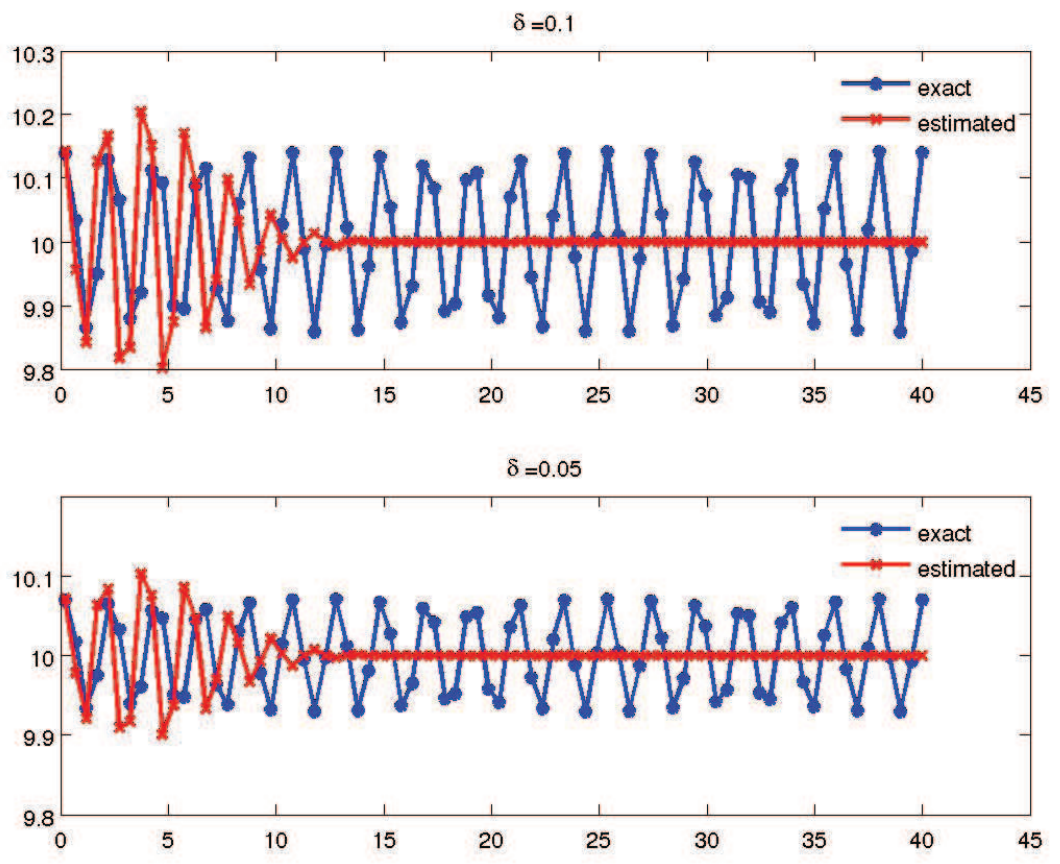

Figure 5: The exact and estimated parameter versus frequencies for $\delta=0.1$ and $\delta=0.05$ for Scheme 2 test function $v_{c s}$ 


\section{REFERENCES}

Anderssen, R. (1999). The pragmatics of solving industrial (real-world) inverse problems with exemplification based on the molecular weight distribution problem. Inverse Problems 15, R1-R40.

Anderssen, R., D. Jackett, and D. Jupp (1984). Linear functionals of the foliage angle distribution as tools to study the structure of plant canopies. Aust. J. Botany 32, 147-156.

Anderssen, R., D. Mead, and J. Driscoll (1997). On the recovery of molecular weight functionals from the double reptation model. JNNFM 68, 291-301. Polymer Melt Rheology Conference, ABERYSTWYTH, WALES, SEP 03-06, 1995.

Anderssen, R., B. Osborne, and I. Wesley (2003). The application of localisation to near infrared calibration and prediction through partial least squares regression. J. NIR Spect. 11, 39-48.

Anderssen, R. S. and M. Hegland (2010). Derivative spectroscopy - an enhanced role for numerical differentiation. JIEA 22, 355-367.

Anderssen, R. S., D. R. Jackett, D. L. B. Jupp, and J. M. Norman (1985). Interpretation of and simple formulas for some key linear functionals of the foliage angle distribution. Agri. Forest Metro. 36, $165-188$.

Bauer, F., P. Mathe, and S. Pereverzev (2007). Local solutions to inverse problems in geodesy. $J$. Geodesy 81, 39-51.

Bear, J. (1972). The Dynamics of Fluids in Porous Media. Elsevier, Amsterdam.

Blakers, R. S., B. F. Kelly, R. S. Anderssen, G. Mariethoz, and W. Timms (2011). 3d dendrogram analysis for mapping aquifer connectivity and flow model structure. Proceedings of MODFLOW and More 2011: Integrated Hydrologic Modeling, Colorado School of Mines, June 5-8, 236-240.

Chow, S.-S. and R. Anderssen (1991). Determination of the tramissivity zonation using linear functional strategy. Inverse Problems 7, 841-851.

Emsellem, Y. and G. de Emarsil (1971). Automatic solution for inverse problem. Water Res. Research 7 , 1264-1283.

Hegland, M. and R. S. Anderssen (2005). Resolution enhancement of spectra using differentiation. Inverse Problems 21(3), 915-934.

Jakeman, A. J. and R. S. Anderssen (1975). Abel type integral-equations in stereology. J. MicroscopyOxford 105, 121-133.

Lamichhane, B. and R. Anderssen (2011). Piecewise constant parameter identification for steady-state diffusion equations. In Preparation.

Lu, S. and S. V. Pereverzev (2009). Sparse recovery by the standard Tikhonov method. Numer. Math. 112, 403-424.

Sun, N. and W. Yeh (1985). Identification of parameter structure in groundwater inverse problem. Water Res. Research 21,869-883.

Yeh, W. (1986). Review of parameter-identification procedures in groundwater hydrology - the inverse problem. Water Res. Research 22, 95-108. 